\title{
Aferição não-invasiva de úlcera por pressão simulada em modelo plano
}

\author{
Non-invasive measurement of pressure ulcer simulated in plain model \\ Evaluación no invasiva de úlcera por presión simulada en modelo llano
}

\author{
Aline Gomes Lopes', Michelle de Campos Soares', \\ Levy Aniceto Santana', Renato da Veiga Guadagnin', Rinaldo de Souza Neves' \\ 'Universidade Católica de Brasília. Brasília , DF
}

Submissão: 22/11/2008

Aprovação: 03/1 2/2008

RESUMO

Estudo Quase-experimental, com o Qual objetivou aperfeiçoar o sistema de projeção dos pontos luminosos de um método já existente, tornando-o portátil, e verificar a acurácia desse novo sistema. O estudo foi realizado por meio do desenvolvimento de um sistema de fotografia digitalizada e processada pela visão computacional em um modelo de superfície plana. Os resultados mostraram erro de I,5 I\% para a profundidade e de - 10,42\% para o volume, concluindo ser um método adequado para aferição de profundidade e volume com erros de acurácia compatíveis com os descritos na literatura.

Descritores: Úlcera de pressão; Cicatrização de feridas; Desenvolvimento experimental.

That is an Quasi-experimental study that aims to improve and make portable an existing method for projecting light points to estimate geometrical properties of a cavity. A computer vision system was developed in order to process input images and calculate volume and depth of a cavity in a plain surface. An error of $1,51 \%$ for depth and $-10,42 \%$ for volume occurred. So this is an adequate method for estimating geometrical properties of cavities with accuracy errors compatible with those errors described in the literature.

Descriptors: Pressure ulcer; Wound healing; Experimental development.

\section{RESUMEN}

Estudio casi-Aferição não-invasiva de úlcera por pressão simulada em modelo planoexperimental, con el objetivo de perfeccionar el sistema de proyección de los puntos luminosos de un método ya existente, volviéndolo portátil, y verificar la acuracia de ese nuevo sistema. El estúdio realizado a través del desarrollo de um sistema de fotografia digitalizada y procesada por la visión computacional en un modelo de superfície llana. Los resultados mostraron um error de 1,51\% para la profundidad y de - 10,42\% para el volumen, concluyendo ser método adecuado para la evaluación de los descriptos em la literatura.

Descriptores: Úlcera por presión; Cicatrización de heridas; Desarrollo experimental. 


\section{INTRODUÇÃO}

As úlceras por pressão são áreas com perda circunscrita de epiderme e derme podendo atingir a hipoderme e tecidos subjacentes decorrentes da associação de vários fatores. Entre eles encontram-se a aplicação de uma alta pressão de curta duração ou uma pressão prolongada ${ }^{(1)}$ causando colapso ou trombose dos vasos capilares, resultando em interferência na oxigenação e nutrição dos tecidos envolvidos, além do acúmulo de subprodutos tóxicos do metabolismo Que levam à anóxia tissular e morte celular ${ }^{(2)}$.

A tolerância tissular - condição da integridade da pele ou das estruturas de suporte - influencia a capacidade do corpo em redistribuir a compressão tecidual contra a estrutura do esQueleto. Esta tolerância tissular reduzida somada a uma pequena área do corpo recebendo uma grande força por pressão gera as chamadas úlceras por pressão ${ }^{(2)}$. Além destes fatores etiológicos existem outros Que predispõem essas lesões, dentre os Quais se encontram a redução do nível de consciência, imobilidade, umidade, fricção e cisalhamento, má nutrição e idade avançada ${ }^{(2)}$.

A maioria das úlceras por pressão ocorre na metade inferior do corpo, sendo Que dois terços correspondem à cintura pélvica, se desenvolvendo em proeminências ósseas e ocorrendo com maior freeüência nas regiões sacra, coccígea, tuberosidade isquiática, trocanteriana, escapular, occipital e maléolos laterais ${ }^{(2)}$.

A investigação e identificação dos fatores de risco determinam a predisposição para formação de úlceras por pressão, devendo-se intervir de forma profilática evitando Que isto ocorra. A melhor opção é escolher a técnica para alívio de pressão (posicionamento adequado no leito, mudanças de decúbito, uso de colchonetes, protetores de calcanhares ${ }^{(3)}$ segundo a efetividade clínica, custo, facilidade de manutenção e conforto do paciente ${ }^{(2)}$.

A fim de orientar a melhor conduta clínica a ser adotada, a NPUAP (National Pressure Ulcer Advisory Panel) classificou as úlceras em Quatro estágios. O estágio I caracteriza-se por um eritema não-esbranQuiçado da pele intacta, Que precede a ulceração; no estágio II ocorre lesão parcial da pele, envolvendo epiderme e/ou derme; no estágio III ocorre lesão total da pele, causando dano ou necrose da camada subcutânea, podendo aprofundar-se, mas não alcançando a fáscia; e no estágio IV ocorre lesão total da pele com grande destruição, necrose tecidual ou danos musculares, ósseos ou de estruturas de suporte ${ }^{(4)}$.

Uma avaliação precisa é capaz de proporcionar o prognóstico das úlceras e estipular a melhor conduta terapêutica. Uma descrição completa inclui a Quantidade de úlceras, a localização, o estágio, o tamanho (comprimento, largura, profundidade), descrição da formação de túneis ou minas, de tecido necrótico, odor ou exsudato e a condição da pele circundante. A melhora pode ser julgada por uma redução do tamanho, do odor, da drenagem, do tecido necrótico e pelo melhor aspecto do tecido de granulação e da pele circunjacente $\mathrm{e}^{(3)}$.

A monitorização pode ser realizada por fotografias seriadas e instrumentos para orientar a avaliação das lesões e Quantificar a cicatrização ${ }^{(3)}$. "Ainda não existem evidências de um instrumento de avaliação de ferida definido como 'padrão ouro' e Que signifieue resultado de cicatrização eficiente”(5). Os métodos mais utilizados e Que são descritos na literatura para mensuração da área, volume (Quantidade de espaço ocupada por um determinado corpo) e profundidade (nível de penetração abaixo de uma superfície) das úlceras podem ser classificados em invasivos e não-invasivos.

Dentre os invasivos, destacam-se métodos capazes de mensurar a profundidade, como a introdução de uma sonda estéril ${ }^{(6: 21)}$ ou uma fina vara ${ }^{(7)}$ até o ponto mais profundo da úlcera; a área, através de modelos de plástico (réguas) $^{(8)}$, filmes plásticos sobre a úlcera (obtendo-se as medidas através de um planímetro, encontrando a área plana) $)^{(8)}$, utilização do aparelho Kundin (mede também volume) ${ }^{(8)}$ e uso de folha de acetato para a obtenção do traçado do perímetro $^{(6)}$; e o volume, pelo preenchimento da úlcera com solução salina Que corresponderá ao volume da ferida ${ }^{(9)}$ ou pelo preenchimento da úlcera por alginato obtendo-se uma réplica da mesma e a medida do volume a partir da massa do alginato ${ }^{(8)}$.

Porém, esses métodos invasivos possuem limitações e desvantagens, tais como: lesão tecidual, risco de contaminação local, infecção de outros pacientes ou da equipe clínica por patógenos do líquido de preenchimento da úlcera ao contaminar a cama e as roupas do paciente; falha no relato de informações a respeito da área, cor e presença de tecido de granulaçãa ${ }^{(8)}$.

As técnicas não-invasivas de mensuração de profundidade, volume, área e cor de úlceras por pressão têm sido desenvolvidas e aprimoradas a fim de reduzir as limitações impostas pelas técnicas invasivas. Técnicas simples de fotografia em preto e branco Que fornecem imagens em duas dimensões (2D) logo evoluíram para imagens coloridas em $2 \mathrm{D}$ possibilitando uma visualização da cor e textura da úlcera ${ }^{(8)}$. Mais recentemente, a associação de fotografia colorida e softwares fornecendo imagens em três dimensões (3D), vem facilitando, assim, a obtenção de dados a respeito da profundidade da úlcera ${ }^{(8)}$.

Os sistemas de mensuração comercializados como o VERG (Vision Engineering Research Group) $^{(8)}$ e o Structured Light $\operatorname{Method}^{(9)}$, são evoluções para obtenção de medidas de úlceras e têm se mostrado mais precisos do que os métodos invasivos.

Outro sistema, Derma, possibilita, além de uma leitura em 3D, a determinação da cor da lesão, e, apesar de seu alto custo e "peso", apresenta-se eficiente já Que possui uma única e uniforme interface para controlar os dados do paciente, apresentar a imagem da úlcera e realizar diferentes medidas e comparações ${ }^{(7)}$.

Um método de varredura a laser para medir a variação do contorno da superfície (topografia) de um objeto sólido foi utilizado para obter o volume e a área de úlceras venosas na perna de 11 indivíduos, demonstrando ser um método útil para mensuração da úlcera ${ }^{(10)}$.

A análise de imagens digitais de uma úlcera de decúbito sacral pelo algoritmo KLT (Karhunen-Loeve Transform) mostrou uma redução da área central da úlcera ao correlacionar os tecidos de granulação e epitelização com duas séries de cores, mostrando-se efetivo apesar de ainda reQuerer validação ${ }^{(11)}$.

A maioria das técnicas não-invasivas apresentam como desvantagens o alto custo, longo período de treinamento do operador do aparelho para obtenção de dados fidedignos e aparelho pesado $^{(8)} \mathrm{e}$ ainda não estão comercialmente disponíveis no Brasil ${ }^{(12)}$.

Estudos vêm sendo desenvolvidos no intuito de reduzir essas desvantagens. Um estudo visando o desenvolvimento de um sistema para mensuração de volume de úlceras por pressão, por meio da visão computacional, na Qual a captação da imagem foi realizada através de uma grade fixada em uma caixa de papelão, projetando 
a imagem dos pontos resultantes da passagem da luz solar em um modelo feito de durepoxi, avaliou o volume de uma úlcera simulada em área plana. A partir da inclinação de 30 graus dos raios solares em relação à normal em um determinado período do dia, foi obtida imagem digital por meio de uma câmera posicionada perpendicular à normal ${ }^{(13)}$. Após processamento da imagem, encontrou-se erro de 12\% da acurácia Quando comparado ao volume real medido com uma pipeta ${ }^{(13)}$. Entretanto esse sistema tem a desvantagem de ser dependente da luz solar e, portanto, inviável na prática clínica.

Por isso, o objetivo do presente estudo foi aperfeiçoar o sistema de projeção dos pontos luminosos de Feistler e Ramos, tornando-o portátil, e verificar a acurácia do volume e profundidade desse novo sistema em uma superfície plana, simulando uma úlcera por pressão.

\section{MATERIAL E MÉTODO}

Foi confeccionado um modelo plano com durepoxi com cavidade simulando a área de uma úlcera por pressão com $5 \mathrm{~cm}$ no maior diâmetro e $4,7 \mathrm{~cm}$ no menor diâmetro.

Para verificação do volume real foi utilizada uma pipeta de 25 $\mathrm{ml}$ da marca Satélit com 0, I ml de precisão e feito o preenchimento total da cavidade com água destilada, sendo Que a Quantidade de líeuido correspondia ao volume da cavidade. A medida foi repetida três vezes, obtendo-se uma média de $9,5 \mathrm{~cm}^{3}$. O cálculo da profundidade máxima real foi obtido com paQuímetro da marca Starret com 0,1 mm de precisão a partir da mensuração da espessura da massa Que preencheu a cavidade, sendo encontrado como profundidade máxima o valor de $8,6 \mathrm{~mm}$, após três tentativas.

Para a produção dos pontos de luz na cavidade do modelo, foi utilizado um tubo de PVC de $100 \mathrm{~mm}$ de diâmetro com $33,5 \mathrm{~cm}$ de comprimento. Foram feitos dois cortes diagonais neste tubo excluindo-se o cone resultante destes cortes. As bordas restantes foram aquecidas e unidas formando o tubo definitivo com 8,5 $\mathrm{cm}$ de diâmetro na extremidade maior e $4 \mathrm{~cm}$ de diâmetro na menor. $\mathrm{O}$ interior do tubo foi pintado de preto para diminuir o reflexo e a perda de luz.

Para produção da luz, foi posicionada dentro do tubo, partindo da extremidade menor, uma caneta laser de $5 \mathrm{~mW}$ com comprimento de onda de $650 \mathrm{~nm} \pm 10$, classe III, utilizando 2 pilhas AAA, com uma lente com $30^{\circ}$ positivos fixada cuidadosamente com supercola (na borda da lente) no emissor do laser, aumentando o feixe de luz $(14 \times 7 \mathrm{~cm})$. O botão da caneta foi mantido ligado com fita isolante. Para a fixação da caneta foram confeccionadas duas circunferências: uma com $4 \mathrm{~cm}$ de diâmetro e outra com $6 \mathrm{~cm}$ de diâmetro, ambas com um orifício por onde a caneta passava.

$\mathrm{Na}$ extremidade maior do tubo foi fixada, com fita isolante, uma tela com 8,5 cm de diâmetro confeccionada no programa Microsoft Office Word 2003 e impressa em transparência, apresentando fundo preto e pontos brancos distantes entre si a $0,4 \mathrm{~cm}$ na vertical e 0,3 $\mathrm{cm}$ na horizontal, tendo sua imagem refletida no modelo após a incidência do laser. Foi limitada, com fita isolante, a área da tela a Qual incidiria suficientemente sobre a cavidade do modelo, sendo o comprimento da linha da imagem projetada de $6,4 \mathrm{~cm} \mathrm{e} \mathrm{o}$ comprimento da coluna de $6,7 \mathrm{~cm}$.

Para a captação da imagem foi utilizada uma máouina fotográfica digital, da marca Canon PowerShot SD450 Digital ELPH 5.0 megapixels, fixada na extremidade maior do tubo por um parafuso de $4 \mathrm{~mm}$ travado com uma porca e uma contraporca e inclinado a $20^{\circ}$ em relação à normal.

O aparelho foi fixado a um tripé e a câmera posicionada perpendicular à normal, sendo isto confirmado por um nível de alumínio, e sobre o modelo a uma distância de $16,5 \mathrm{~cm}$, medida por uma régua simples, permitindo o enQuadramento e visualização adequada da imagem. A imagem foi registrada em um ambiente sem luminosidade para melhor visualização dos pontos.

\section{Processamento da Imagem em Programação lava}

Primeiramente foi realizado o acinzentamento da imagem com opção de critério pelo Qual ocorre a transformação da tonalidade colorida de cada ponto, definida por valores distintos dos componentes vermelho, verde e azul $(\mathrm{R}, \mathrm{G}, \mathrm{B})$, no intervalo de 0 a 255, em uma tonalidade cinza com valor único para esses componentes baseada no componente vermelho.

A próxima etapa foi a limiarização dinâmica pela Qual houve a transformação da tonalidade cinza, com valores variando de zero (preto) a 255 (branco), para valores zero ou 255, conforme a faixa à Qual pertence à tonalidade, a saber, abaixo ou acima, de um valor prefixado. $\mathrm{O}$ valor desse limiar foi definido com variação descendente conforme a distância do ponto em Questão, até o ponto central da imagem. Esse procedimento se tornou necessário para compensar a maior iluminação da parte central do modelo promovida pelo dispositivo de iluminação utilizado. Em seguida, as tonalidades obtidas foram invertidas com a finalidade de melhor visualização, resultando em orifícios escuros e fundo branco.

Foram ainda aplicados procedimentos de contração, para eliminação de pequenos objetos isolados e de expansão, para retorno ao tamanho inicial dos objetos.

\section{Cálculo do Volume}

A imagem foi segmentada em objetos correspondentes aos orifícios e, logo após, foram determinadas as coordenadas dos centros de massa ou centróides de cada objeto. Retas foram definidas unindo os centros de massa dos objetos das bordas da imagem, definindo, assim, a localização dos pontos da superfície fora da cavidade.

Foi realizado o cálculo das distâncias (d) entre os centros de massa de todos os objetos e as retas ligando os centros de massa dos objetos das bordas da imagem, obtendo, assim, os desvios dos raios provocados pela cavidade, ou seja, a definição das faixas de área correspondente a cada ponto.

Cálculo da Profundidade Correspondente a cada Orifício (h)

Foi obtida através da divisão da distância entre o centro de massa e a reta ligando os centros de massa dos objetos das bordas da imagem, pela tangente do ângulo de inclinação do feixe de raios em relação à direção vertical (20 graus).

$$
\mathrm{h}=\mathrm{d} / \operatorname{tg} 20^{\circ}
$$

\section{Cálculo da Área Correspondente a cada Orifício (s)}

Foi determinada a área correspondente à Quantidade de pontos abrangida por cada objeto da imagem, correspondente à área real, com base em uma escala. No presente estudo, obteve-se 23,67 $\mathrm{mm}^{2}$ de área unitária. 


\section{Cálculo do Volume da Cavidade do Modelo (v)}

O volume foi calculado por meio da multiplicação da soma de todas as profundidades pela área correspondente a cada orifício.

$$
\mathrm{v}=(\Sigma \mathrm{h}) \times \mathrm{s}
$$

\section{RESULTADOS}

Pelo método computacional foram encontrados $8,51 \mathrm{~cm}^{3}$ para o volume e $8,73 \mathrm{~mm}$ para profundidade a partir das medições de cada ponto da imagem obtida.

A comparação da medida real com a computacional mostrou um erro da acurácia para o cálculo da profundidade de 1,51\% e para o cálculo do volume de 10,42\% para baixo, havendo uma diferença de $0,99 \mathrm{~cm}^{3}$ entre o volume real e o calculado pela visão computacional.

\section{DISCUSSÃO}

O valor de erro da acurácia para o cálculo da profundidade não pôde ser comparado com a literatura, pois não foram encontrados relatos de estudos Que tenham calculado essa acurácia em outros métodos. No entanto, fazendo um paralelo com um estudo similar ao presente, porém utilizando superfície convexa, encontrou-se um erro menor, pois o estudo anterior ${ }^{(12)}$ havia encontrado erro de $4,44 \%$ para a profundidade.

Os achados do atual estudo mostraram um erro maior de acurácia para o cálculo do volume, porém ainda menor Que o encontrado por Feistler e Ramos ${ }^{(13)}$ Que descreveram erro de $12 \%$, estando, entretanto, na faixa de erro descrita por Plassmann et al ${ }^{(9)}$ Que, utilizando um sistema de luz estruturada, descreveram erros entre 3 e $15 \%$.

Além disso, neste estudo foi possível criar um sistema portátil, possibilitando seu transporte e manuseio; aprimorar a luz mudando a fonte da mesma; reduzir a distância entre os pontos da imagem projetada permitindo medidas e cálculos mais precisos; e com baixo custo totalizando aproximadamente US\$ 17,00 dólares, excluindo a câmera digital, o tripé e o computador, enquanto outros estudos relatam valores em torno de US\$2.600,00 dólares ${ }^{(9)}$ e US\$ 51 1.000,00 dólare $S^{(7)}$. O software ICreator ,de programação lava utilizado, está disponível na internet a custo zero.

\section{CONSIDERAÇÕES FINAIS}

O método, aperfeiçoado e agora portátil, mostrou-se adequado para aferição de profundidade e volume com erros de acurácia compatíveis com os descritos na literatura, porém seu uso ainda não é viável na prática clínica.

Sugerem-se Que estudos futuros melhorem a acurácia desse sistema e viabilizem seu uso em aplicações clínicas à luz ambiente mas Que mantenham o baixo custo para Que possa ser acessível a todos profissionais na avaliação da efetividade dos recursos fisioterapêuticos e de enfermagem no tratamento dessas lesões.

Para tanto, propõe-se a utilização de outros instrumentos para obtenção de medidas reais de volume; aplicação do sistema de projeção dos pontos luminosos em diferentes tipos de modelos; obtenção da área real da cavidade do modelo, verificando se a mesma interfere na medida do volume; e aproximação dos orifícios da tela, gerando dados mais precisos.

\section{REFERÊNCIAS}

1. Kottke FJ, Lehmann JF. Tratado de medicina física e reabilitação de Krusen. $4^{\text {a }}$ ed. São Paulo: Manole; 1994.

2. Jorge SA, Dantas SRPE. Abordagem multiprofissional do tratamento de feridas. São Paulo: Atheneu; 2005

3. Duthie EH, Katz PR. Geriatria Prática. $3^{\text {a }}$ ed. Rio de Janeiro: Revinter; 2002.

4. Bergstrom N. Treatment of pressure ulcers. Clinical practice guideline number 15. Washington: U.S. Departament of Health and Human Services. Public Health Service. Agency for Health Care Policy and Research. Publication No 95-0652; 1994.

5. Bork AMT. Enfermagem baseada em evidências. Rio de Janeiro: Guanabara Koogan; 2005.

6. Posso MBS. Semiologia e semiotécnica de enfermagem. Atheneu: São Paulo; 2006.

7. Callieri M, Cignoni P, Pingi P, Scopigno R. Derma: monitoring the evolution of skin lesions with a 3D system. VMV 2003; 666.

8. Krouskop TA, Barker R, Wilson MS. A noncontact wound

measurement system. J Rehab Res Develop 2002; 39: 337-46.

9. Plassmann P, Melhish JM, Harding KG. Methods of measuring wound size - a comparative study. Wounds 1994; 6:54-61.

10. Marjanovic D, Dugdale RE, Vowden P, Vowden KR. Measurement of the volume of a leg ulcer using a laser scanner. Phisiol Meas 1998; 19: 535-43.

11. Souza MGP, Quintiliano P, Trindade L, Santana L, Sá E, Guadagnin R. Recognition of texture and area of decubitus ulcers through computer vision. Pattern Recog Image Anal 2005; 15 : 586-88.

12. Marques KC, Santos LPL. Validação de um método nãoinvasivo e de baixo custo para avaliação de volume e profundidade de úlceras por pressão em superfície convexa [monografia]. Brasília (DF): Universidade Católica de Brasília, Curso de Fisioterapia; 2005.

13. Feistler FR, Ramos RCN. Validação de um método de avaliação do volume de escaras [monografia]. Brasília (DF): , Universidade Católica de Brasília, Curso de Fisioterapia; 2004. 